\title{
Bilateral Exertional Compartment Syndrome With Endoscopic Fasciotomy Surgical Intervention in a High School Athlete
}

\author{
Madeleine C. Gwinn ${ }^{1}$, Aaron McGuffin ${ }^{1}$ \\ 1. Pediatrics, West Virginia School of Osteopathic Medicine, Lewisburg, USA
}

Corresponding author: Madeleine C. Gwinn, mgwinn@osteo.wvsom.edu

\begin{abstract}
A 17-year-old female presented to the physical therapy clinic with bilateral lower leg pain that worsened with activity. The patient experienced numbness, tingling, and cramping along the lateral and posterior portions of her legs during basketball practice, and her symptoms had gradually worsened over the past eight months. She obtained minimal relief with conservative treatments such as stretching and rest. X-rays and MRI of the lower limbs were obtained six months after symptoms began and were unremarkable. Further evaluation included compartment pressure testing taken before and after exercise. The patient demonstrated diagnostic pressures indicative of compartment syndrome in two compartments bilaterally. The patient was subsequently diagnosed with exertional compartment syndrome.
\end{abstract}

Exertional compartment syndrome is a cause of muscle pain that occurs due to increased tissue pressure within the confinement of the closed fascial space during exercise. Patients are often misdiagnosed or there is a significant delay in the correct diagnosis. The gold standard for diagnosis is measurement of intracompartmental pressures with the Stryker catheter.

Clinicians should consider exertional compartment syndrome in active patients who present with generalized muscle pain or sensation deficits that worsen with activity and are relieved with rest. Surgical intervention is a reasonable intervention and the only definitive option for an athlete with chronic exertional compartment syndrome who wants to continue high-level competition. Endoscopic fasciotomies are the new preferred techniques compared to more invasive open surgeries, which require a full incision. Endoscopic fasciotomy has a quicker healing time and has been shown to be as effective at relieving compartment syndrome symptoms as invasive open techniques.

After surgical intervention, the patient reported a $90 \%$ reduction in symptoms and had returned to full sport participation within two months.

Review began 01/15/2021 Review ended 02/09/2021 Published 02/13/2021

\section{๑) Copyright 2021}

Gwinn et al. This is an open access article distributed under the terms of the Creative Commons Attribution License CC-BY 4.0., which permits unrestricted use, distribution, and reproduction in any medium, provided the original author and source are credited.
Categories: Pediatrics, Physical Medicine \& Rehabilitation, Orthopedics

Keywords: bilateral compartment syndrome, endoscopic fasciotomy, exertional compartment syndrome

\section{Introduction}

Compartment syndrome is a well-known condition requiring immediate attention due to increased intracompartmental pressures, which can lead to vascular compromise and limb ischemia. However, exertional compartment syndrome is less widely known and often an overlooked diagnosis. Consequently, this patient case serves as an important learning tool to improve recognition of the symptoms associated with chronic exertional compartment syndrome and the surgical interventions available for resolution. We will discuss the case of a 17-year-old female athlete whose symptoms were significantly limiting her athletic performance. We will also discuss the steps that were taken to diagnosis and treat her condition.

This study was previously presented as a poster at the WVSOM (West Virginia School of Osteopathic Medicine) Alumni Association Mid-Winter Conference, January 25, 2020, Charleston, West Virginia, USA.

\section{Case Presentation}

A healthy 17-year-old female presented to the clinic with bilateral lower leg pain that worsened with activity. Symptoms began as a gradual aching and cramping along the lateral and posterior portions of her legs. Increased weight-bearing exercises, specifically running, caused increased symptoms including sensation deficits in her lower legs. Symptoms progressed to the point where the patient was unable to play continuously. She required several non-weight-bearing breaks and lower extremity stretching to continue with activity. At the height of her symptoms, post-exercise, her physical examination revealed decreased dorsiflexion, generalized weakness, and decreased sensation in the lateral and posterior lower leg. Gait analysis showed a "tip toe gait" with decreased stride length and absent heel strike during deceleration. Posterior tibial and dorsalis pedis pulses were normal. Sensation returned to normal as soon as the patient 


\section{Cureus}

became non-weight-bearing. Early intervention included stretching, phonophoresis ultrasound with $1 \%$ dexamethasone, bilateral orthotic shoe placement, NSAIDs, and heat/ice therapy. However, all conservative interventions failed to relieve symptoms during exercise.

\section{Diagnostic methods}

X-rays of her tibia, fibula, and ankle mortise along with an MRI of her bilateral lower limbs were unremarkable. Further diagnostic studies included compartment pressure testing. Pressures were taken 10 minutes prior to running on a treadmill and immediately afterwards.

Table 1 summarizes the compartment pressure testing pre- and post-exercise. The patient met the diagnostic criteria in two compartments pre-exercise and in four compartments post-exercise.

\begin{tabular}{|l|l|l|}
\hline Compartment & Pressure Readings $(\mathbf{m m H g})$ Pre-Exercise & Pressure Readings (mmHg) Post-Exercise \\
\hline Right anterior & 9 & 25 \\
\hline Right lateral & 20 & 9 \\
\hline Right posterior superficial & 2 & 8 \\
\hline Right posterior deep & 7 & 28 \\
\hline Left anterior & 11 & 25 \\
\hline Left lateral & 18 & 4 \\
\hline Left posterior Superficial & 0 & 7
\end{tabular}

TABLE 1: Compartment testing results pre- and post-exercise

A resting pressure of $15 \mathrm{mmHg}$ and post exercise pressure $20 \mathrm{mmHg}$ are diagnostic of chronic compartment syndrome [1].

\section{Discussion}

The waxing and waning presentation with activity makes the diagnosis of chronic compartment syndrome difficult. The diagnosis relies heavily on the patient history and timing of symptoms, which adds to the complexity of the diagnosis. Classical symptoms are pain in the affected compartment at the same time, distance, or intensity of exercise [2]. Typical diagnosis of chronic compartment syndrome has a 22-month delay [3]. Other differentials are more common and require less invasive diagnostics. These include medial tibial stress syndrome, stress fractures, other nerve entrapment syndromes, and deep vein thrombosis. However, if there is strong suspicion for chronic exertional compartment syndrome, the diagnosis should be pursued.

The lower leg is divided into four compartments: anterior, lateral, superficial posterior, and deep posterior. The tibialis posterior muscle is contained within its own fascia and is considered the fifth compartment of the leg [3].

Figure 1 operation imaging demonstrates the anterior compartment and the lateral compartment before and after the surgical fasciotomy. 


\section{Cureus}
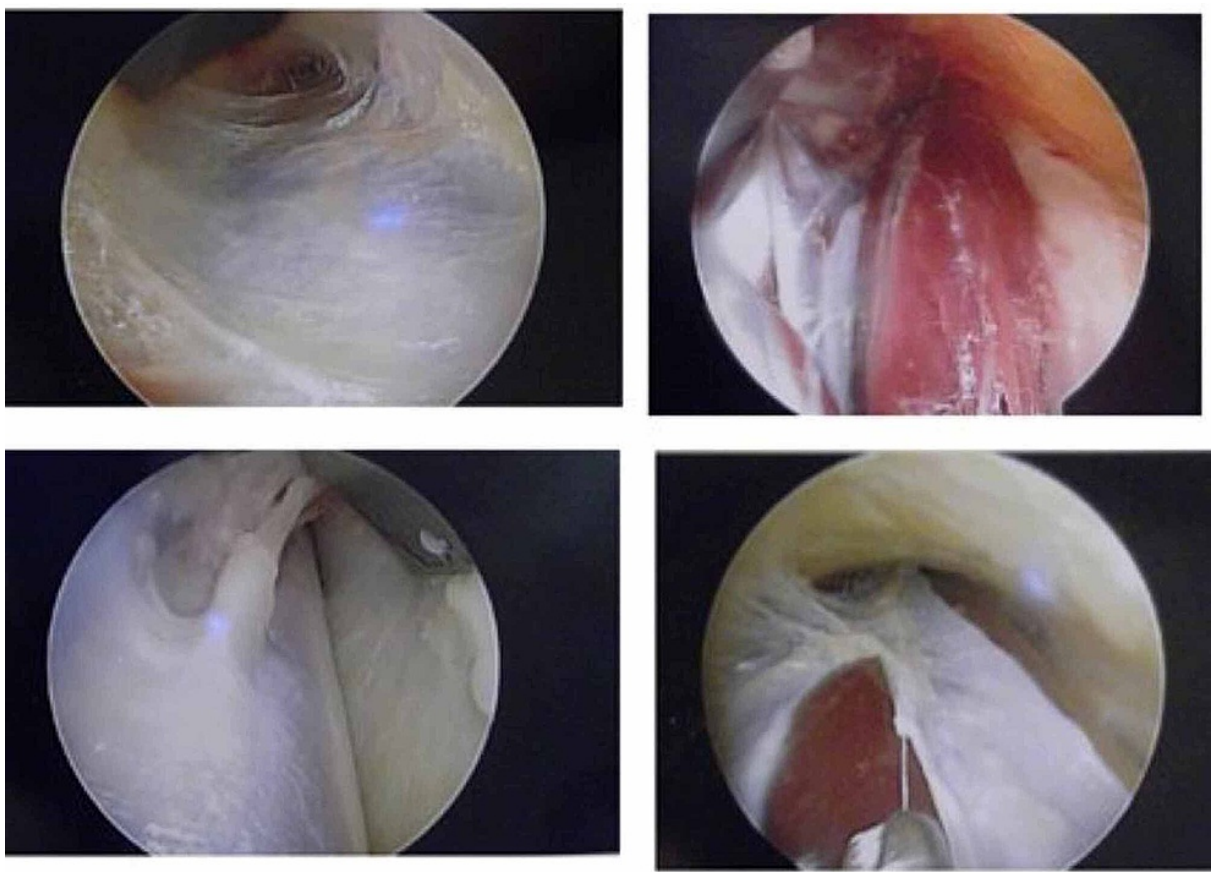

FIGURE 1: Top left: anterior compartment before fasciotomy. Top right: anterior compartment after fasciotomy. Bottom left: lateral compartment before fasciotomy. Bottom right: lateral compartment during fascia release.

Exertional compartment syndrome is a cause of muscle pain that occurs due to increased tissue pressure within the confinement of a closed fascial space during exercise [2]. The muscle volume can increase up to $20 \%$ of its resting size during exercise. This increase in muscle volume increases the internal pressure within the fascial compartment [1]. The pain caused by this increase in internal pressure is thought to be related to impaired tissue perfusion [4]. The gold standard of diagnosing chronic exertional compartment syndrome is the measurement of intracompartmental pressures. The Stryker catheter is the most widely used instrument for pressure testing. The procedure includes placing a needle inside one of the four compartments and injecting normal saline into that compartment. The pressure of the compartment is then taken and measured in $\mathrm{mmHg}[2]$.

The patient elected to undergo bilateral endoscopic fasciotomy to the anterior, lateral, and posterior compartments. Endoscopic fasciotomy is a subcutaneous technique that is less invasive than open fasciotomy. This technique is favored due to the smaller incision and quicker healing time [5]. Anterior and lateral fasciotomies have the best outcome with a greater than $80 \%$ success rate [6]. Overall complication of this intervention is $11-13 \%$ and includes hemorrhage, infection, nerve damage, deep vein thrombosis, vascular injury, skin breakdown, lymphocele, altered sensation over the fasciotomy site, and nerve entrapment [7].

Figure 2 illustrates the small incisions that were used for the endoscopic procedure. 


\section{Cureus}

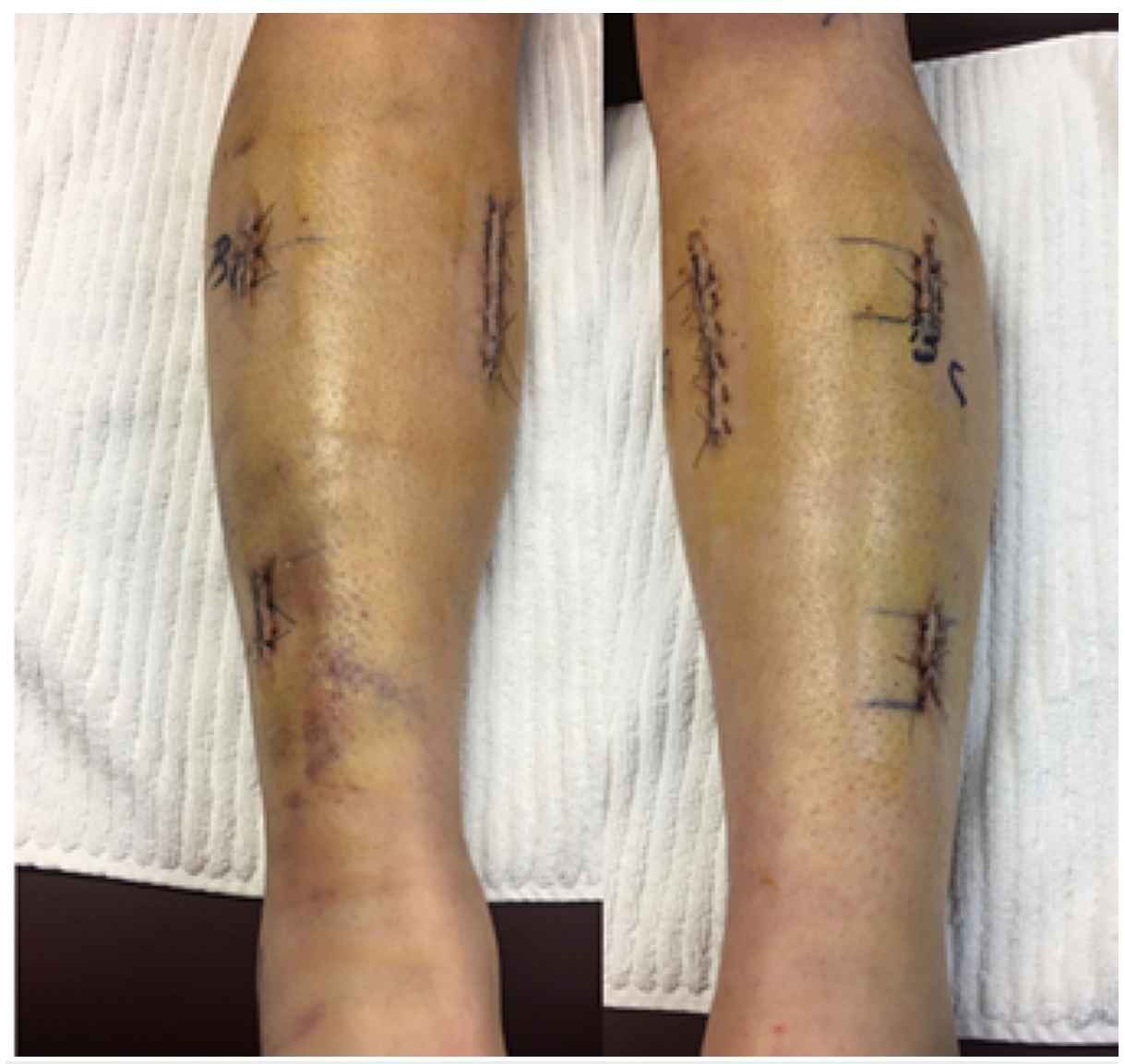

FIGURE 2: Patient incisions 24 hours after surgery.

\section{Conclusions}

Once the incisions were healed, the patient began a return to play protocol, which included a mobility and strengthening plan. Within two months of surgery, she returned to full participation in basketball, no longer exhibited gait abnormalities, and reported $90 \%$ resolution of symptoms.

Exertional compartment syndrome should be considered in the differential when an active patient presents with generalized muscle pain or sensation deficits that are relieved by rest. MRI and X-rays should be completed to rule out other etiologies. Compartment pressure testing is the gold standard for the diagnosis of chronic exertional compartment syndrome. A surgical fasciotomy is commonly the treatment of choice because conservative treatments often do not fully resolve symptoms. An athlete can return to full activity in six to eight weeks if symptom-free and have recovered full strength and flexibility, making this an ideal treatment option.

\section{Additional Information}

\section{Disclosures}

Human subjects: Consent was obtained or waived by all participants in this study. Conflicts of interest: In compliance with the ICMJE uniform disclosure form, all authors declare the following: Payment/services info: All authors have declared that no financial support was received from any organization for the submitted work. Financial relationships: All authors have declared that they have no financial relationships at present or within the previous three years with any organizations that might have an interest in the submitted work. Other relationships: All authors have declared that there are no other relationships or activities that could appear to have influenced the submitted work.

\section{References}

1. Canale ST, Beaty JH, Campbell WC: Campbell's Operative Orthopaedics. Elsevier, Philadelphia, PA; 1998

2. Tucker AK: Chronic exertional compartment syndrome of the leg. Curr Rev Musculoskelet Med. 2010, 3:3237. 10.1007/s12178-010-9065-4

3. Frontera WR, Silver JK, Rizzo TD: Essentials of Physical Medicine and Rehabilitation. Elsevier, Philadelphia, PA; 2002.

4. Goldfarb S, Kaeding C: Bilateral acute-on-chronic exertional lateral compartment syndrome of the leg: a 


\section{Cureus}

case report and review of the literature. Clin J Sports Med. 1997, 7:59-62. 10.1097/00042752-19970100000011

5. Due J Jr, Nordstrand K: A simple technique for subcutaneous fasciotomy . Acta Chir Scand. 1987, 153:521522.

6. DeLee JC, Drez D Jr, Miller MD: DeLee and Drez's Orthopaedic Sports Medicine. Elsevier, Philadelphia, PA; 2003.

7. Howard JL, Mohtadi NG, Wiley JP: Evaluation of outcomes in patients following surgical treatment of chronic exertional compartment syndrome in the leg. Clin J Sports Med. 2000, 10:176-184. 10.1097/00042752-200007000-00005 